\title{
Dopant Imaging of Power Semiconductor Device Cross Sections
}

\author{
U. Gysin ${ }^{\text {a }}$, E. Meyer a, Th. Glatzel ${ }^{\text {a }}$, G. Günzburger ${ }^{\text {b }}$, H. R. Rossmann, ${ }^{\text {c }}$, T. A. Jung ${ }^{\text {acc }}$, \\ S. Reshanov ${ }^{\mathrm{d}}$, A. Schöner ${ }^{\mathrm{d}}, \mathrm{H}$. Bartolf ${ }^{\mathrm{e}}$ \\ ${ }^{a}$ Department of Physics, University of Basel, 4056 Basel, Switzerland \\ ${ }^{\mathrm{b}}$ Helmholtz-Zentrum Berlin, Hahn-Meitner-Platz 1, 14109 Berlin, Germany \\ ${ }^{c}$ Laboratory for Micro- and Nanotechnology, Paul Scherrer Institute, 5232 Villigen PSI, \\ Switzerland \\ d Ascatron AB, Electrum 207, 16440 Kista, Sweden \\ e ABB Switzerland Ltd, Corporate Research, 5405 Baden-Dättwil, Switzerland \\ e-mail: ernst.meyer@unibas.ch
}

Keywords: Dopant Imaging, Scanning Probe Microscopy (SPM), Power Semiconductor Devices, Silicon Carbide

\begin{abstract}
Several Scanning Probe Microscopy (SPM) methods allow to image dopant profiles in a range from $10^{14} \mathrm{~cm}^{-3}$ to $10^{19} \mathrm{~cm}^{-3}$ on semiconducting samples. In our work we present Scanning Capacitance Force Microscopy (SCFM) and Kelvin Probe Force Microscopy (KPFM) experiments performed on cross sections of silicon $(\mathrm{Si})$ and silicon carbide $(\mathrm{SiC})$ power devices and epitaxially grown calibration layers. The KPFM signals show under illumination a reduced influence on surface defect states. In addition results from numerical simulation of these microscope methods are discussed.
\end{abstract}

\section{Introduction}

The characterization of power electronic device cross-sections is an essential part of manufacturing and optimization processes. One of the most important information is the mapping of the targeted dopant concentrations, since they determine the electronic performance of the device. It is found that Secondary Ion Mass Spectroscopy (SIMS) is difficult to be applied in case of power semiconductor devices because of the low dopant concentrations (as low as $10^{14} \mathrm{~cm}^{-3}$ ) and narrow dimensions in the $\mu \mathrm{m}$ range. In this contribution, a dedicated Scanning Probe Microscope (SPM) [1], operated under ultra-high vacuum conditions, is applied for this purpose. An image of the scanning probe microscope instrument is shown in Fig. 1. The most important operation modes are Scanning Spreading Resistance Microscopy (SSRM) [2], Kelvin Probe Force Microscopy (KPFM) [3] and Scanning Capacitance Force Microscopy (SCFM) [4]. On one hand, SSRM is found to be a robust method for silicon samples. This SPM technique measures the local spreading resistance at relatively high loading forces. The main disadvantage is the destructive nature of this imaging mode, where high normal forces of about $50 \mu \mathrm{N}$ are required to penetrate through the native oxide layer of the semiconductor surface. On the other hand, KPFM and SCFM are non-contact methods, which are sensitive enough to observe dopant concentrations in the range of $10^{14} \mathrm{~cm}^{-3}$ to $10^{19} \mathrm{~cm}^{-3}$. KPFM is found to be influenced by surface defects [5], which can lead to partial pinning of the Fermi level and subsequently a reduced contact potential difference (CPD) is observed. The band bending due to defects can be reduced by suitable sub-bandgap illumination of the tip-surface interface [6]. 
a)

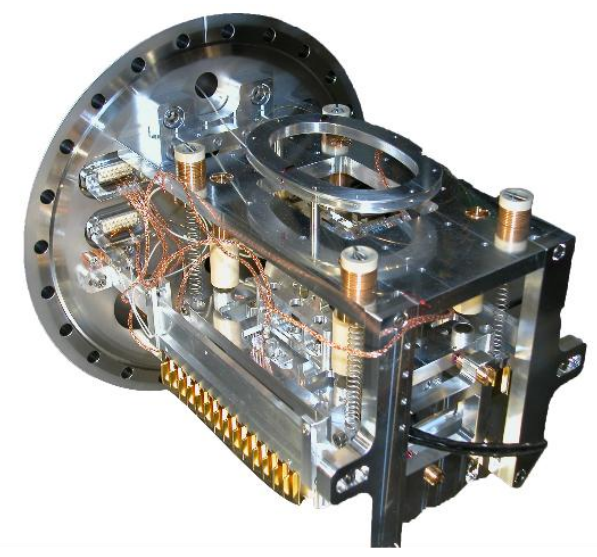

b)

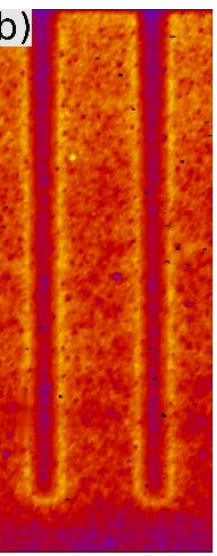

$-160$

$$
\mathrm{V}_{\mathrm{CPD}}[\mathrm{mV}]
$$

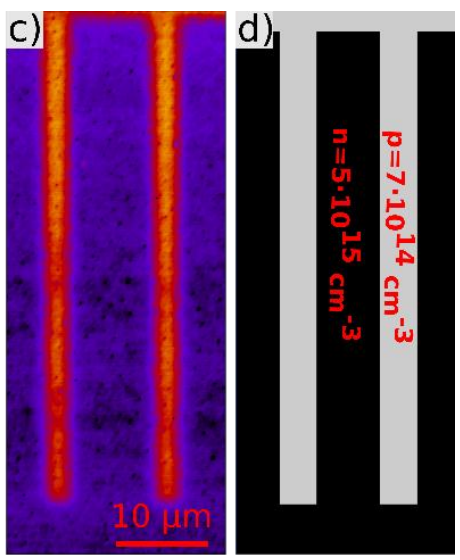

Figure 1: (a) AFM with a 100x100 $\mathrm{m}^{2}$ closed loop scanner operating in UHV conditions with full optical access [1]. KPFM images of a silicon test structure (b) without and (c) with irradiation (about 20mW, $\lambda=475$ $525 \mathrm{~nm}$ ) by sub-bandgap photons, respectively. The p-doped trenches with approximate concentrations of 5 $7 \cdot 10^{14} \mathrm{~cm}^{-3}$ are surrounded by the n-type substrate as shown schematically in $(d)$.

\section{Experimental Results}

To demonstrate the capability of our instrument, we performed dopant imaging on Si-based high-voltage super-junction device structures [7]. These structures were manufactured by a demanding and complex trench-etching followed by epitaxial refill process. The trenches have a depth of $60 \mu \mathrm{m}$, a width of $3 \mu \mathrm{m}$ and are Boron-doped with concentrations in the range of $10^{14}-10^{15} \mathrm{~cm}^{-3}$ to get p-type conductivity. We found that SSRM provides valuable quantitative information about the doping level by comparison of the measured data at the region of interest to previously calibrated epitaxially grown layers [8]. Furthermore, the trenches were investigated by the non-contact method, KPFM, as shown in Figs. 1 b) and c). KPFM measures the contrast in the CPD signal by applying a bias voltage $\mathrm{V}_{\mathrm{CPD}}$ to compensate electrostatic forces, which is sensitive to defect structures at the surface. A decrease of the defect density by suitable sample preparation and the reduction of the surface band bending by illumination with sub-bandgap irradiation are feasible strategies to minimize band bending and to optimize the CPD contrast mechanism $[9,10]$. SCFM seems to be a valuable method to visualize differently doped regions, but still needs further sophisticated modelling to obtain quantitative results.
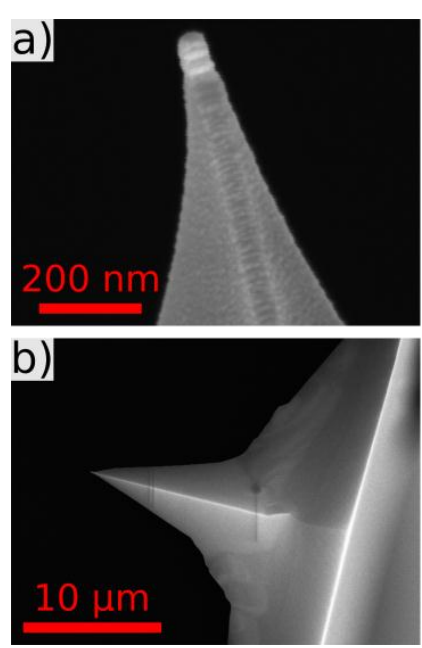

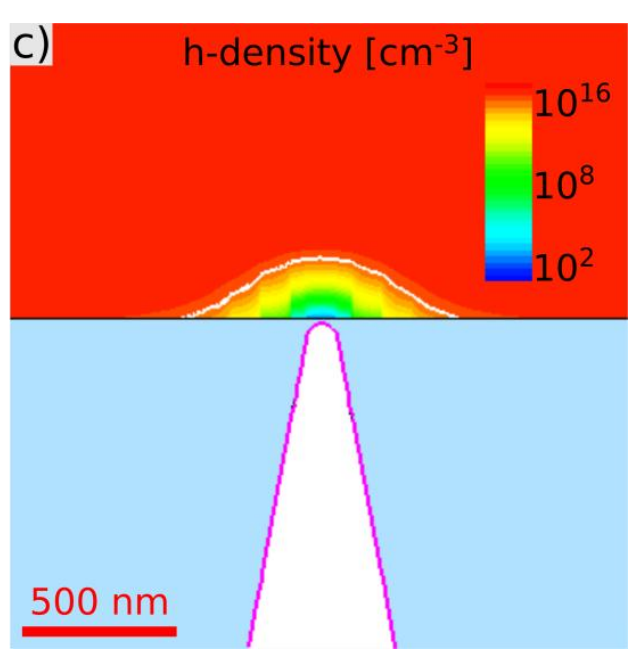

Figure 2: (a) and (b) SEM images of a Pt/Ir coated AFM tip used for KPFM and SCFM measurements. (a) exhibits the radius of the tip apex of approximately 50nm. (c) Synopsys Sentaurus TCAD simulation of the hole density in the vicinity of the metallic probing tip on a silicon surface. The bulk density is $p=10^{16} \mathrm{~cm}^{-3}$. The depleted zone at an applied voltage of $5.8 \mathrm{~V}$ extends about $200 \mathrm{~nm}$ into the semiconductor resulting in a measurable capacitance by the SCFM approach. 
Figure 2 c) shows a Synopsys Sentaurus TCAD simulation of the hole density in the vicinity of a probing metallic tip on a silicon surface. The depletion zone below the probing tip leads to a change of the capacitance as a function of dopant density. The radius of curvature of the probing tips is about $50 \mathrm{~nm}$ as shown in the corresponding SEM pictures in Figs. 2 a) and b). In addition, SiC-device structures were investigated with the above mentioned methods. In this case, SSRM was found to be difficult to be applied because of the extreme hardness of SiC (close to diamond), which makes it difficult to form a stable electronic contact between the diamondcoated tip and the SiC surface.

\subsection{KPFM Method}

Kelvin probe force microscopy (KPFM) relies on the measurement of electrostatic forces between probing tip and sample as a function of applied voltage $\mathrm{V}_{\mathrm{CPD}}$ [3]. For compensated contact potential, the electrostatic force is at its minimum. Ideally, the contact potential differences (CPD) is influenced by the shift of the Fermi energy,

$$
V_{C P D}=\frac{1}{q}\left(\chi+\frac{E_{g}}{2}+k_{B} T \cdot \ln \left(\frac{N_{A}}{n_{i}}\right)-\phi_{m}\right)
$$

for $p$-doping and

$$
V_{C P D}=\frac{1}{q}\left(\chi+\frac{E_{g}}{2}-k_{B} T \cdot \ln \left(\frac{N_{D}}{n_{i}}\right)-\phi_{m}\right)
$$

for $n$-doping, where $\chi$ is the electron affinity, $E_{\mathrm{g}}$ is the band gap, $k_{\mathrm{B}}$ is the Boltzmann constant, $T$ the temperature, $q$ the elementary charge, $n_{\mathrm{i}}$ the intrinsic carrier density, $\phi_{\mathrm{m}}$ the work function of the metallic tip and $N_{\mathrm{D}}$ is the donor density and $N_{\mathrm{A}}$ the acceptor density. However, the Fermi level can be influenced by interface trap states or surface defects, which leads to band bending and a reduction or increase of the CPD for $\mathrm{p}$ - and n-type semiconductors, respectively. In extreme cases, the Fermi level is pinned and the CPD would be independent of dopant concentration. The application of sub-band gap irradiation can reduce the influence of the band bending. This results in CPD values which are closer to the theoretical expectations. As shown in the KPFM measurement of a Si trench structure presented in Fig. 1 b) the contrast between $n$ - and $p$-doped regions can even be inverted (large CPD on $n$-doped areas and smaller CPD on $p$-doped area). Application of light irradiation yields the expected contrast due to the reduction of band bending (Fig. $1 \mathrm{c})$ ).

\subsection{SCFM Method}

Scanning capacitance force microscopy (SCFM) uses force detection to probe the local capacitance. In contrast to scanning capacitance microscopy (SCM), which measures local capacitances by electrical means, SCFM is based on purely mechanical detection by measuring amplitude and phase of the second resonance frequency of the cantilever. Typically, resonance frequencies of the order of $1 \mathrm{MHz}$ are used, which means that the method is based on the fast response of the capacitance. It is well known from MIS-capacitance measurements, that the AC-capacitance gives only changes due to accumulation and weak depletion. However, inversion is not observable due to the finite time response of the minority carriers. Similarly, we do observe signals due to accumulation and depletion, where the change of the capacitance due to the depletion capacitance gives the largest signal. As shown in the calculated capacitance gradient vs. tip bias curves presented in Fig. 3, the strongest SCFM signal is observed on the areas with lowly doped concentrations, where the depletion boundary locally penetrates deepest into the semiconductor and the depletion capacitance is smallest, giving rise to large changes 


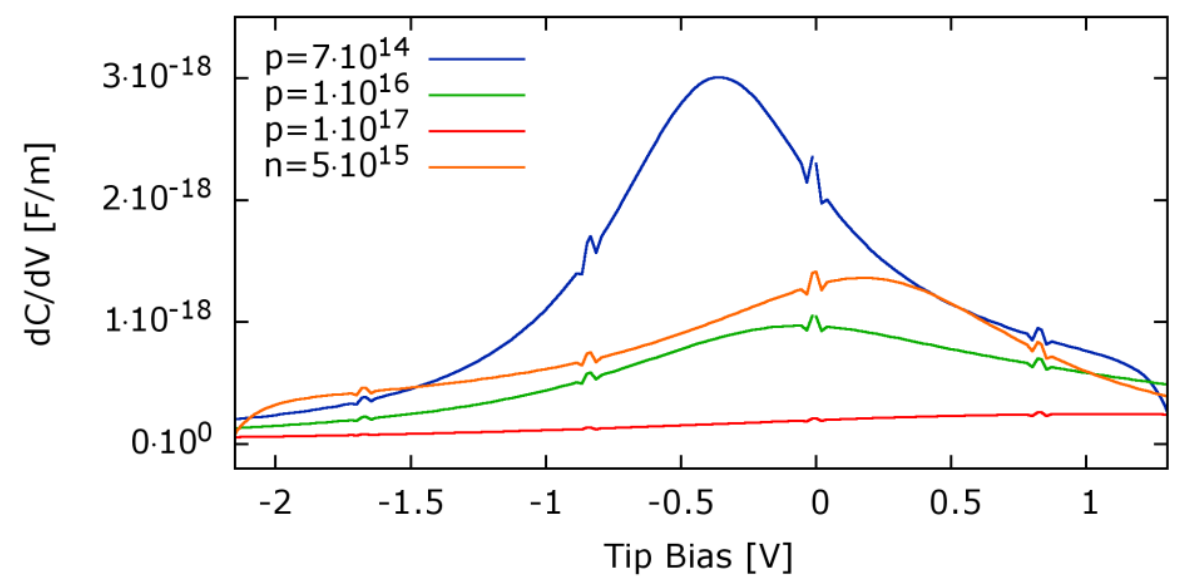

Figure 3: Calculated capacitance gradient for a $50 \mathrm{~nm}$ tip at $10 \mathrm{~nm}$ separation above a silicon surface for different doping concentrations. Interestingly, the biggest gradient is observed for the smallest dopant density which makes SCFM an ideal candidate for dopant imaging with high sensitivity in the low-doping range which is difficult to access by conventional methods (e.g., SIMS).

of the total capacitance. Simulations with Synopsys Sentaurus TCAD, taking into account the realistic geometry of the cantilever and probing tip (radius of curvature $50 \mathrm{~nm}$ and opening angle of $10^{\circ}$ ), show reasonable agreement between the SCFM measurements on silicon layers. The small ripples of the curves are due to numerical artefacts. Clearly, the largest amplitude is observed for the lowest dopant concentration of $7 \cdot 10^{14} \mathrm{~cm}^{-3}$. Subsequently, smaller signals are measured for $1 \cdot 10^{16}$ and $1 \cdot 10^{17} \mathrm{~cm}^{-3}$. SCFM results in a relatively weak contrast for highly doped regions, because the total capacitance is not varying as much during the transition through the depletion region. The contrast of the $n$-region is mirrored at the $y$-axis, but shows similar trends with the largest contrast on the lowly doped areas. A comparison between KPFM and SCFM on cross sections of SiC calibration layers shows dopant dependent contrasts, where concentrations between $10^{15} \mathrm{~cm}^{-3}$ and $10^{20} \mathrm{~cm}^{-3}$ can be distinguished, cf. Fig. 4. The amplitude $\mathrm{A}_{2}$ of the third harmonic oscillation tuned to the second mechanical Eigenmode of the cantilever

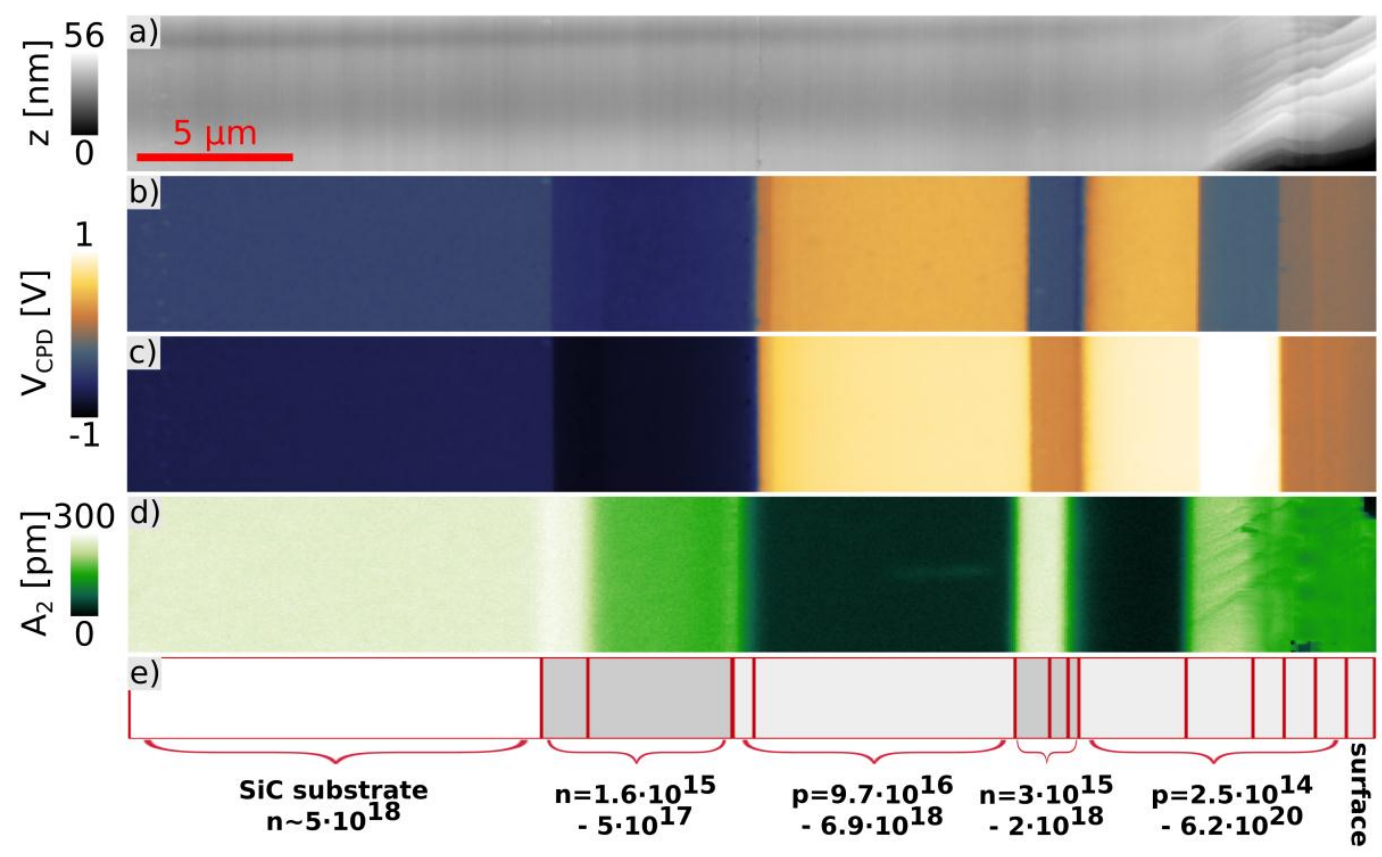

Figure 4: (a) Topography (b) KPFM in dark (c) KPFM with laser irradiation (about 10mW, $\lambda=450-500 \mathrm{~nm}$ ) and (d) SCFM on well-defined SiC calibration layers. Bright contrast in SCFM corresponds to the weakest dopant concentrations of $10^{15} \mathrm{~cm}^{-3}$. A schematic of the differently doped regions is shown in (e). 
is related to the voltage derivative of the force gradient by $A_{2} \propto \frac{\partial^{2} C}{\partial V \partial z} V_{A C}^{3} \sin (3 \omega t)$, where $\mathrm{V}_{\mathrm{AC}}$ is the drive voltage of the electric excitation.

\section{Conclusion}

Dopant imaging of cross-sections of semiconductor power devices has been successfully demonstrated for silicon and silicon carbide devices. In the case of silicon, SSRM is found to be a reliable technique. SSRM is based on the application of large normal forces in a range of $50 \mu \mathrm{N}$, forming an ohmic-like contact between the SPM tip and the sample. In the case of silicon carbide, SSRM is not suitable due to the hardness of SiC. Here, the non-contact methods KPFM and SCFM provide the desired information, but are significantly affected by defect states. Sub-bandgap laser irradiation reduces the band bending and provides improved CPD contrast. SCFM gives the strongest contrast for regions with low doping concentrations, where the reduction of the total capacitance due to the wide depletion layer is the strongest.

\section{Acknowledgements}

This work was supported in part by the Swiss National Science Foundation and the Nano Argovia program of the Swiss Nanoscience Institute.

\section{References}

[1] U. Gysin, T. Glatzel, T. Schmölzer, A. Schöner, S. Reshanov, H. Bartolf and E. Meyer, "Large area scanning probe microscope in ultra-high vacuum demonstrated for electrostatic force measurements on high-voltage devices", Beilstein Journal of Nanotechnology 6, 24852497, (2015).

[2] P. De Wolf, J. Snauwaert, T. Clarysse, W. Vandervorst and L. Hellemans, "Characterization of a point-contact on silicon using force microscopy-supported resistance measurements”, Appl. Phys. Lett. 66, 1530, (1995).

[3] M. Nonnenmacher, M. P. O'Boyle and H. K. Wickramasinghe, "Kelvin probe force microscopy”, Appl. Phys. Lett. 58, 2921, (1991).

[4] K. Kobayashi, H. Yamada and K. Matsushige, "Dopant profiling on semiconducting sample by scanning capacitance force microscopy", Appl. Phys. Lett. 81, 2629-2631, (2002).

[5] C. Sommerhalter, T. Matthes, T. Glatzel, A. Jäger-Waldau and M. C. Lux-Steiner, "Highsensitivity quantitative Kelvin probe microscopy by noncontact ultra-high-vacuum atomic force microscopy", Appl. Phys. Lett. 75, 286, (1999).

[6] T. Glatzel, S. Sadewasser, R. Shikler, Y. Rosenwaks and M. Lux-Steiner, "Kelvin probe force microscopy on III--V semiconductors: the effect of surface defects on the local work function", Mat. Sci. Engineer. B 102, 138, (2003).

[7] H. Bartolf, A. Mihaila, I. Nistor, M. Jurisch, B. Leibold, M. Zimmermann, "Development of a $60 \mu \mathrm{m}$ Deep Trench and Refill Process for Manufacturing Si-Based High-Voltage SuperJunction Structures,'IEEE Transactions On Semiconductor Manufacturing, vol. 26, no. 4, pp. 529-541, (2013).

[8] H. Bartolf et al, Proc. ISPSD 2015, Hong-Kong, pp. 281-284 (2015).

[9] H. R. Rossmann, U. Gysin, A. Bubendorf, T. Glatzel, S. Reshanov, A. Schöner, T. A. Jung, E. Meyer, H. Bartolf, "Two-Dimensional Carrier Profiling on Lightly Doped n-type 4HSiC Epitaxially Grown Layers,” Mat. Sci. For., Vols 821-823 (2015), pp 269-272. 
[10] L. Kronik and Y. Shapira, "Surface photovoltage phenomena: theory, experiment, and applications”, Surf. Sci. Rep. 37, 1-206, (1999). 

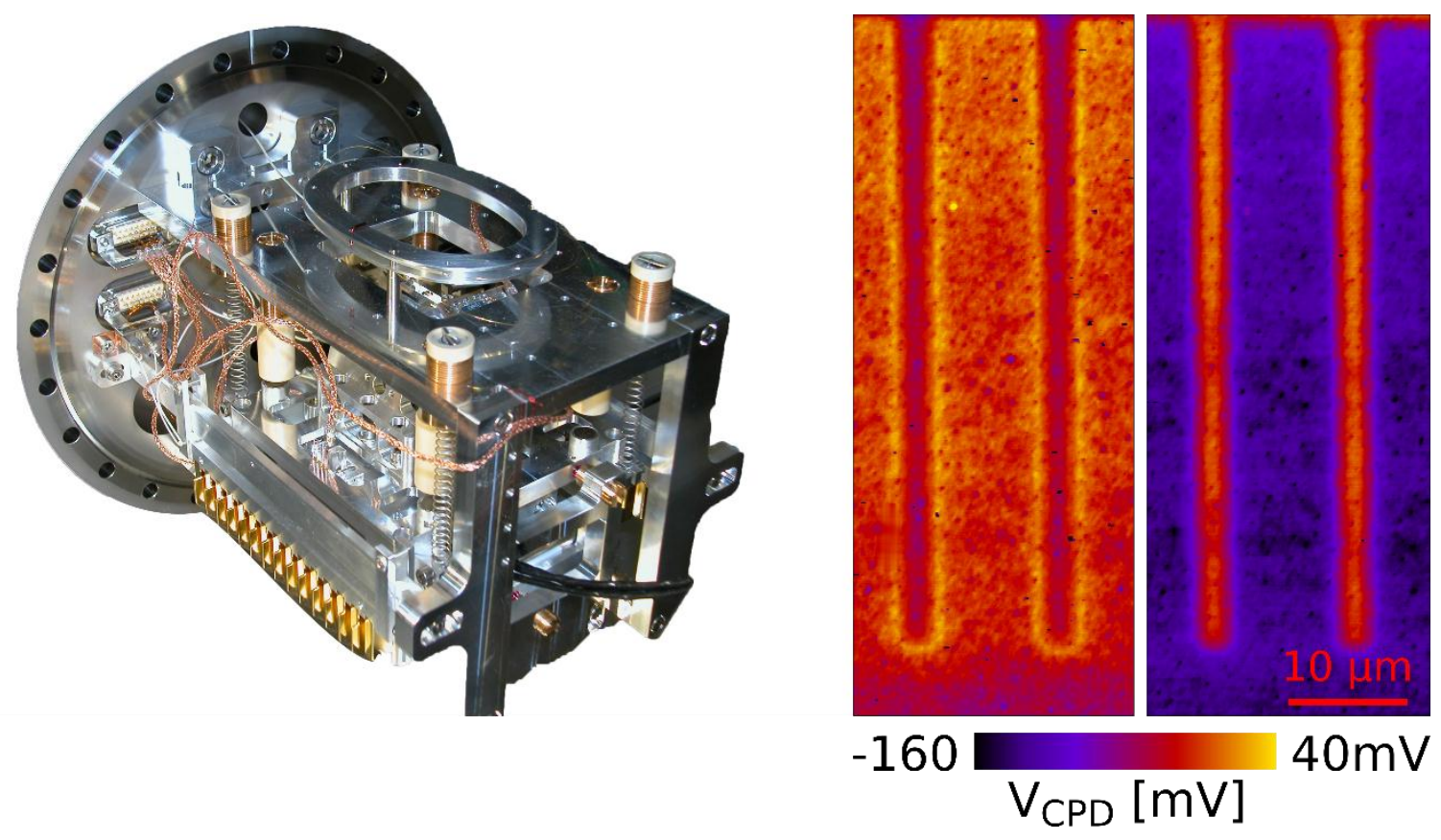